\title{
DESIGN OF THE SNS ACCUMULATOR RING VACUUM SYSTEMS*
}

\author{
H.C. Hseuh", C.J. Liaw, M. Mapes, BNL, Upton, NY 11973
}

\section{Abstract}

Brookhaven is undertaking the design, construction and commissioning of the accumulator ring and the beam transport lines for the 1-GeV Spallation Neutron Source. The operating pressure of the accumulator ring vacuum system will be $10^{-9}$ Torr and is needed to minimize the beam-residual gas ionization. The vacuum requirement, the layout of the ring vacuum system, and the design of the vacuum chambers, vacuum pumps and other hardware are presented here.

\section{INTRODUCTION}

The goal of the Spallation Neutron Source (SNS)[1] is to provide a short pulse $(\sim 0.5 \mu \mathrm{s})$ of proton beam with an average beam power of $1 \mathrm{MW}$ to a neutron generating target. This is to be achieved by a $1-\mathrm{GeV}$ linac and accumulator ring operated at $60 \mathrm{~Hz}$ with $1 \times 10^{+14}$ protons per pulse. To improve reliability and to allow hands-on maintenance, the radiation level caused by uncontrolled particle loss in the ring during one msec period must be less than $10^{-4}$ per pulse. There are several vacuum related beam loss mechanisms, which are nuclear scattering, multi-Coulomb scattering and residual gas ionization.

The nuclear scattering cross sections, $\sigma_{n}$, are proportional to the geometrical cross section of the target nucleus and are $\sim 4 * 10^{-25} \mathrm{~cm}^{2}, \sim 3 * 10^{-25}$ and $\sim 4 * 10^{-26} \mathrm{~cm}^{2}$ for $\mathrm{CO}, \mathrm{H}_{2} \mathrm{O}$ and $\mathrm{H}_{2}$ respectively. These gasses are the most common residual gasses in a clean ultrahigh vacuum system. The beam loss due to nuclear scattering is given by $\Delta \mathrm{I} / \mathrm{I}=\int \beta c \sigma_{n} \mathrm{~N}$ dt with $\mathrm{N}$ being the residual gas density in the vacuum system. At the designed pressure of $1 \times 10^{-9}$ Torr, $\mathrm{N}$ will be $6 \times 10^{+7}$ atoms $/ \mathrm{cm}^{3}$. The resultant $\Delta \mathrm{I} / \mathrm{I}$ will be $<10^{-9}$ over one msec.

The multi-Coulomb scattering causes growth of the RMS beam size. The fraction of the increase in beam size can be calculated by [2] $\Delta \sigma_{y} / \sigma_{y}=\int k \beta_{y} N /\left(p^{2} \varepsilon\right) \mathrm{dt}$, with $\sigma_{\mathrm{y}}$ the transverse beam dimension, $k=1.085 \times 10^{-23}$ $(\mathrm{GeV} / \mathrm{c})^{2} \mathrm{~m}^{3} \sec ^{-1}, \beta_{y}$ the betatron amplitude $(<20 \mathrm{~m}), p$ the momentum $(=1.696 \mathrm{GeV} / \mathrm{c})$ and $\varepsilon$ the transverse emittance $(<240 \pi \mathrm{mmmrad})$. The fraction of beam growth over one millisecond will be $<10^{-8}$ at the design pressure of $1 \times 10^{-9}$ Torr.

The $e-p$ instability can limit the achievable intensity [3] when excessive electrons are generated and trapped. *Work performed under the auspices of the U.S. Department of Energy
"Email: hseuh@ bnl.gov
Therefore, the production of electrons due to beamresidual gas ionization must be minimized. The ionization cross sections $\sigma_{i}$ of the $1 \mathrm{GeV}$ protons on residual gas molecules can be calculated using Bethe formula [4]:

$$
\sigma_{i}=4 \pi\left(\frac{h}{m_{e} c}\right)^{2}\left(M^{2}\left[\frac{1}{\beta^{2}} \ln \left(\frac{\beta^{2}}{1-\beta^{2}}\right)-1\right]+\frac{C}{\beta^{2}}\right)
$$

with $4 \pi\left(h / m_{e} c\right)^{2}=1.87 \times 10^{-20} \mathrm{~cm}^{2}$, and $M^{2}$ and $C$ the experimentally determined coefficients for different molecules. With a residual gas composition of $40 \% \mathrm{H}_{2}$, $40 \% \mathrm{H}_{2} \mathrm{O}$ and $20 \% \mathrm{CO}$ in the accumulator ring, the average $\sigma_{i}$ for $1 \mathrm{GeV}$ proton $(\beta=0.875)$ will be $\sim 6 \times 10^{-19}$ $\mathrm{cm}^{2}$. At the designed vacuum of $1 \times 10^{-9}$ Torr, the rate of electron production is given by:

$$
\frac{d n_{e}}{d t}=\beta N \sigma_{i} n(t)
$$

with $n(t)$ being the instantaneous number of protons in the ring. Integration and averaging of the above equation over a one-millisecond cycle gives $\sim 3 \times 10^{-4}$ electrons per proton which is negligible [3] in comparison with the production of the electrons from other mechanisms.

\section{RING VACUUM LAYOUT}

The accumulator ring, with a circumference of 220 $\mathrm{m}$ has four arc regions and four long straight regions (a four-fold symmetric lattice). The vacuum system is divided into eight vacuum sectors, four arc vacuum sectors and four straight vacuum sectors, isolated with allmetal pneumatic gate valves. The arc vacuum sectors are $\sim 32 \mathrm{~m}$ long, consisting of eight half-cell vacuum chambers which are 4 meters long. The straight vacuum sectors are $\sim 23 \mathrm{~m}$ long, consisting of four quarter-cell chambers as well as special chambers for injection, collimation, RF, instrumentation and extraction.

Metals and ceramics are used exclusively in the construction of the vacuum system since they are UHV compatible and radiation resistant. No elastomer or organic materials are allowed. Stainless steel, which has excellent mechanical/vacuum properties and is easy to use during fabrication, has been selected as the material for the half-cell and quarter-cell chambers. Conflat-type flanges and seals will be used to join the chambers together. In several locations with potentially high background radiation, such as the injection, extraction and collimator regions, quick-disconnect type flanges and seals will be used, which will minimize the radiation exposure during machine maintenance periods. All chambers will be chemically cleaned and assembled in a 
clean environment to minimize contamination.

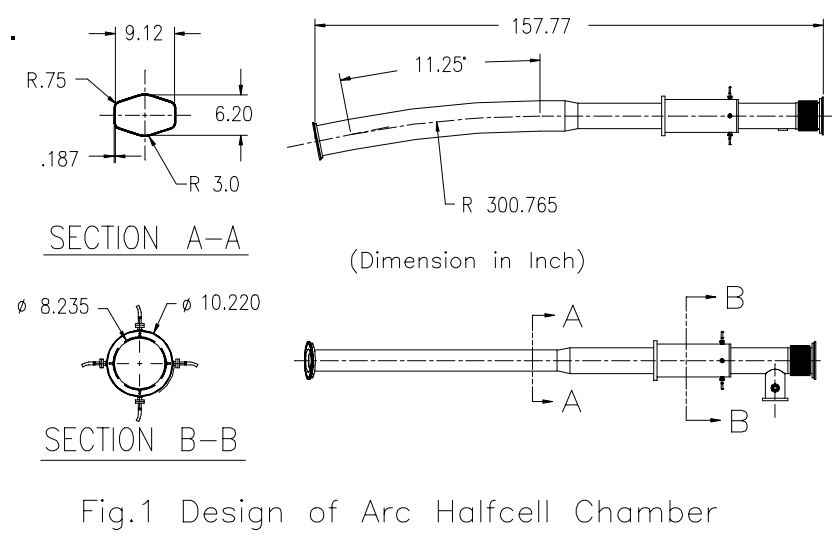

\subsection{Half-cell Chambers}

Fig. 1 shows a standard arc half-cell chamber. The two-meter long dipole chamber will have an elliptical inside cross section of $23 \mathrm{~cm}(\mathrm{H})$ by $16 \mathrm{~cm}(\mathrm{~V})$, providing ample aperture for future upgrade to $2 \mathrm{MW}$. The chamber will be curved with a bending angle of 11.25 degrees and fabricated from 300 series stainless steel. The top and bottom halves of the dipole chamber will be formed by rolling stainless steel sheets on a curved die. The two halves will then be TIG welded together along the mid plane. To minimize the deflection and to assure the structural stability of the chamber under vacuum load, the wall thickness of the dipole sections will be $\sim 5 \mathrm{~mm}$. Results of the stress, deformation and buckling analysis, using ANSYS code, are given in Fig.2.

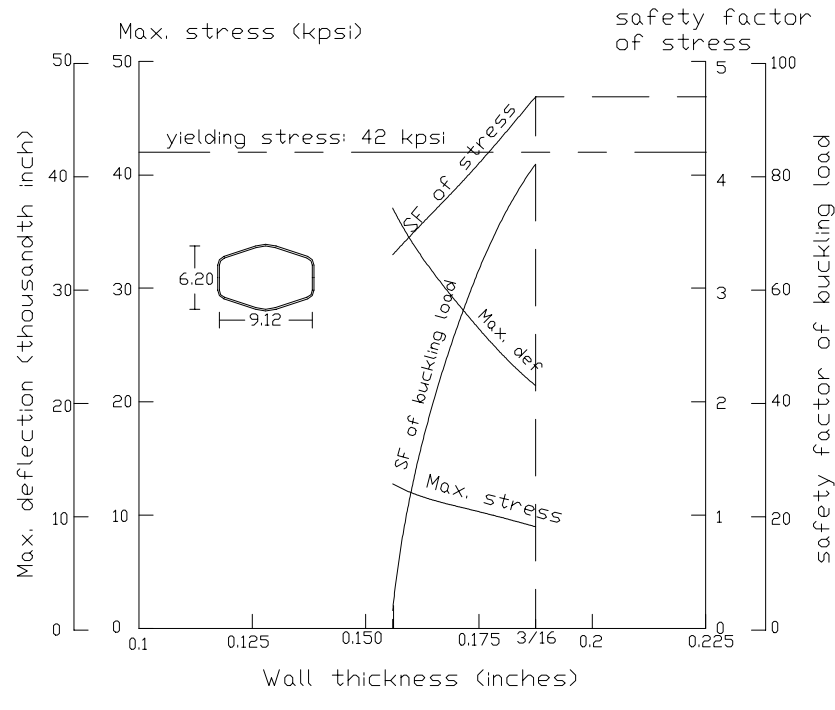

Fio.2: Structural Analysis of SNS Dipole Chamber

With the selected wall thickness and a safety factor of 4.5 , the maximum deflection of the chamber is $\sim 0.5 \mathrm{~mm}$. The safety factor for the buckling is $\sim 90$. To reduce wall impedance, there will be a tapered transition from the dipole chamber to the round quadrupole pipe. The quadrupole pipe has an I.D. of $19 \mathrm{~cm}$ and will be seamless stainless steel tubing. The remainder of the half-cell chamber consists of a beam position monitor (BPM), a pump port and a bellows. The stripline-type BPM is $0.5 \mathrm{~m}$ long and is to be precisely aligned with respect to the quadrupole magnet.

To minimize distortion, the BPM housing will be copper brazed to a rigid connecting flange which is then TIG welded to the quadrupole pipe. The quadrupole-BPM section will then be joined to the dipole section with an automated welder on a precision fixture. The short pump section contains the bellows and the pump port and will be tapered to match the elliptical cross section of the adjacent dipole chamber. To minimize the radiation induced stress corrosion, the thin wall bellows will be fabricated from Inconel. The pump port which has a $203 \mathrm{~mm}$ Conflat flange will be screened with $>80 \%$ transparency for evacuation. Side ports with $70 \mathrm{~mm}$ Conflat flanges will be provided above the pump port for other vacuum hardware such as vacuum gauges, residual gas analyzers and roughing valves. The pump section and the end Conflat flanges will be TIG welded to the dipole/quadrupole chamber. The completed half-cell chambers will be wrapped with a thin layer of Kapton insulation before installation into the 'opened' magnets.

\subsection{The Straight Section Chambers}

Each straight vacuum sector will have four quartercell chambers and the special chambers housing collimators, RF cavities and injecton and extraction equipment. The quarter-cell chamber consists of a quadrupole pipe, BPM, pump port, bellows and flanges and will be made of stainless steel. The fabrication of the quarter-cell chambers will be similar to that of the halfcell chambers.

\section{VACUUM PERFORMANCE}

The eight ring vacuum sectors will be isolated with all-metal gate valves. Vacuum sensors (i.e., vacuum gauge and ion pump readings) upstream and downstream of the gate valves will interlock the valves protecting the ring from catastrophic vacuum failures. The valves also allow repair and modification of components without venting other regions to ambient air. Turbomolecular pump stations backed by dry mechanical pumps will be used to pump down the vacuum sectors and allow the high vacuum pumps to be conditioned and activated. The high linear conductance provided by the large aperture of the half-cell and quarter-cell chambers makes distributed pumping unnecessary. Lumped pumps will be used. One sputter ion pump of $\sim 200 l / \mathrm{sec}$ will be installed on each chamber. In addition, titanium sublimation pumps of $500 l / \mathrm{sec}$ each, which are housed inside the sputter ion pump, will be used to compliment the sputter ion pumps. The net pumping speed at the pump port will exceed 500 $l / \mathrm{sec}$.

The thoroughly cleaned stainless steel chambers will have an outgassing rate of $\sim 1 \times 10^{-11}$ Torr. $l / \mathrm{sec} . \mathrm{cm}^{2}$, a few 
days after pump down from atmosphere. The pressure inside the chambers can be calculated by:

$$
P(x)=Q\left(\frac{L}{S}+\frac{L^{2}-x^{2}}{2 C^{\prime}}\right)
$$

with $Q$ being the outgassing per linear length $\left(\sim 1 \times 10^{-9}\right.$ Torr. $l / \mathrm{sec} . \mathrm{cm}), 2 L$ the distance between pumps $(2 L=400$ $\mathrm{cm}$ in the arc sectors), $2 S$ the pumping speed at the neck of the pump $(2 S=500 \mathrm{l} / \mathrm{s}), C^{\prime}$ the linear conductance of the chamber $\left(\sim 3 \times 10^{5} l . \mathrm{cm} / \mathrm{sec}\right.$ for $\mathrm{H}_{2}, \sim 1 \times 10^{5} l . \mathrm{cm} / \mathrm{sec}$ for $\mathrm{H}_{2} \mathrm{O}$ and $\sim 8 \times 10^{4} l . \mathrm{cm} / \mathrm{sec}$ for $\mathrm{CO}$ ), and $x$ the distance to the nearby pump ( $x=0$ at the pump and $x=L$ halfway between the pumps). The pressure distribution in the halfcell chamber with no beam is shown in Fig. 3. The average pressure will be $\sim 7 \times 10^{-10}$ Torr.

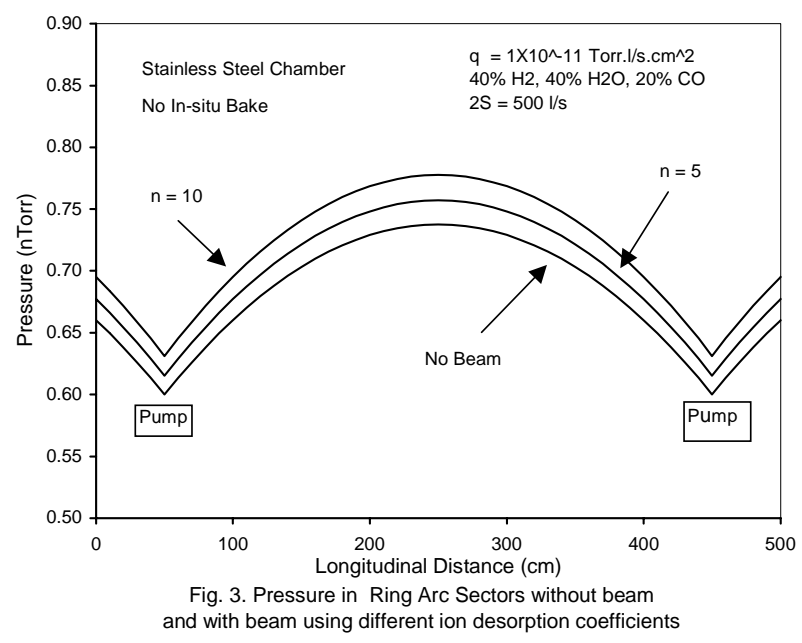

With beam present, protons will ionize the residual gas molecules. These new ions, repelled by the beam-wall potential, will bombard the chamber wall and desorb molecules. Each ion with $\sim \mathrm{keV}$ energy [5] will desorb 5 molecules from the unbaked stainless steel surface [6]. The equilibrium pressure in the chamber with the beam will then be given by [7]:

$$
P(I, x)=\frac{Q}{C^{\prime} W^{2}}\left[\frac{\cos W x}{\cos W L-\frac{W C^{\prime}}{S} \sin W L}-1\right]
$$

where $\mathrm{W}^{2}=I \sigma_{\mathrm{i}} \eta / C^{\prime}, I$ being the average proton intensity over the 16-millisecond cycles, and $\eta$ being the average number of molecules desorbed from the chamber wall by an incident ion. The pressure distribution inside the half-cell chambers is plotted for $\eta=5$ and $\eta=10$ in Fig. 3. Due to the large linear conductance of the chambers and the high pumping speed available, the pressure increase due to ion desorption is less than $10 \%$ even for $\eta=10$. No pressure bump instability is expected. The pumping requirement in the four straight regions will be modeled with the detailed design of these regions. The designed vacuum will be achieved by positioning several large pumps adjacent to the high outgassing components such as the extraction kickers.

\section{VACUUM INSTRUMENTATION}

Two sets of pirani and cold cathode gauges (CCGs) will be installed in each vacuum sector, covering the pressure range from 1000 Torr down to $10^{-3}$ Torr, and from $10^{-3}$ Torr to $10^{-10}$ Torr, respectively. The sputter ion pumps will be powered by $5 \mathrm{KV}$ controllers, which are capable of reading current down to sub microampere levels. The pump current, proportional to pressure, will give a more detailed pressure profile around the ring. All gauges, residual gas analyzers, controllers and power supplies are commercially available. Due to the radiation level in the tunnel, all electronics including controllers and power supplies will be located in the ring support building. All controllers will have remote capabilities in addition to front panel switches. They will communicate with the vacuum programmable logic controllers (PLCs) through RS-485 type serial links for remote monitoring and control. The PLCs with PC-based LabView type application software will provide the interlock logic and on-line menu for the operation of the gate valves. An ethernet-type link will connect the PLC to the main control console for monitoring, logging and control of the vacuum devices. In addition, hardwired interlocks will connect the PLCs to the beam permit chassis and abort the beam in the event of a vacuum fault.

\section{SUMMARY}

There are unique features in the design of the SNS accumulator ring vacuum system. To avoid the e-p instability due to beam-residual gas ionization, ultrahigh vacuum of $1 \times 10^{-9}$ Torr is required. This will be achieved by using UHV compatible materials, processes, and providing sufficient pumping from the combination of sputter ion pumps and titanium sublimation pumps. To minimize radiation exposure during maintenance periods, large quick-disconnect flanges will be used. All vacuum instruments will be capable of remote operation.

\section{REFERENCES}

[1] W.T. Weng, 'Accumulator Ring Design for SNS Project', PAC'97, Vancouver, May, 1997.

[2] G. Guignard, CERN 77-10, p33, June 1977.

[3] Ruggiero, A.G. and Blaskiewicz, M., BNL/SNS Tech. Note \#8, January, 1997.

[4] Baconnier,Y., Proc. CERN Accel. School, CERN 85-19, pp267300, Nov. 1985.

[5] Hseuh, H.C., BNL/SNS Tech. Note \#15, February, 1997.

[6] R. Calder and A.G. Mathewson, Vacuum, 29, 53(1979).

[7] Fischer, E. and Zankel, K., CERN-ISR-VA/73-52, Nov. 1973. 